\title{
A Bystander Behaviour Model in bullying situations
}

\author{
Yuriko Isada $^{1}$, Nobuko Igaki ${ }^{2},{ }^{*}$ and Aiko Shibata ${ }^{3}$ \\ ${ }^{1,2}$ Kwansei Gakuin University, Japan \\ ${ }^{3}$ International Christian University, Japan
}

\begin{abstract}
.
In this paper, the behaviour of bystanders is analysed. It is said that the structure of bullying consists of three parties, namely "bullies", "victims" and "bystanders" and that it is important to control bullying by changing bystanders' behaviour. In general, bystanders may act like they don't see bullying even if they witness it. However, an individual bystander may take action if more bystanders take action to stop bullying. Conversely, when most bystanders act to prevent bullying, some may think that they don't have to act. A mathematical model is formulated assuming that the action timings are different for each person. In more concrete, we suppose that each bystander is influenced by the ratio of members who take action to stop bullying. We call this ratio the active rate. Each bystander has his/her own lower threshold and his/her own upper threshold, then, if the active rate is between these two thresholds he/she takes action to stop bullying. That is, each bystander knows the active rate every day and decides whether to act or not to act on the following day. Simulations are performed for this model. As a result, it is found that the existence of highly motivated members has a positive effect on the entire bystanders. On the other hand, even if there are no highly motivated members, it could be quite effective to continue educating members with low motivation.
\end{abstract}

Keywords: bystander, bullying, collective behaviour, mathematical model, simulation

\section{Introduction}

The Act on Promotion for the Measures to Prevent Bullying was enacted in 2013 (MEXT, 2013), as an effect of a suicide of a junior high school student due to bullying occurred in the previous year in Otsu City in Japan. As serious bullying situations were stipulated by Article 28, Paragraph 1 of the Act, the survey on problems in guidance of students' behaviour was conducted all over Japan. It was shown that the number of serious bullying situations occurred totalled 79 incidents at 158 schools in 2013 (MEXT, 2014) and 444 incidents at 474 schools in 2017(MEXT, 2018). Thus, the survey indicates no sign of a decrease in the serious situation of bullying.

Morita (2010) analyses the structure of bullying and indicates that "bystanders" exist other than "bullies" and "victims" as the parties to the bullying. He pointed out that it is important to control bullying by changing "bystanders" to "moderators".

In this study, we examine a mathematical model that considers the action of bystanders' behaviour where bullying continues. This paper comprises as follows. Section 2 describes our 
20-22 March, 2020

\section{Berlin, Germany}

\section{$10^{\text {th }}$ International Conference on Humanities, Psychology \& Social Sciences}

model in detail. Simulation experiment and its analysis are shown in section 3 . Section 4 discusses changes in bystander behaviour due to educational policies. Our conclusions are presented in Section 5.

\section{The active zone model}

The model dealt with in this paper is a model of bystander behaviour in a classroom where bullying continues. In the classroom, we analyse the behaviour of these $n$ bystanders, assuming that there are bullies, victims, and $\mathrm{n}$ bystanders. Bystanders may take action to deter bullying or may pretend not to notice. The more people who take action to stop bullying, the more they will take action to stop bullying, but the criteria for taking action will be different depending on each individual. Some people may take action if $20 \%$ of them take action, and others may take no action even if $80 \%$ of them take action. With this in mind, in this paper, we assume that each person has a unique threshold for taking action.

Conversely, when most people act to prevent bullying, some may think that they don't have to act. Considering such a phenomenon called a so-called free rider, we assume that there is another threshold (upper threshold) depending on each individual for stopping action. In other words, suppose that each person has two thresholds (lower threshold and upper threshold).

Let us formulate this situation to a mathematical model. First, the ratio $X(t)$ of bystanders who took action to stop bullying on the day $t$ is called the active rate. Each bystander knows the active rate $X(t)$ every day and decides whether to act or not to act on the following day. Bystander i has lower threshold $L_{i}(t)$ and upper threshold $U_{i}(t)$. We call interval $\left[L_{i}(t), U_{i}(t)\right]$ active zone. If the active rate $X(t)$ is in his/her active zone, he/she acts on the following day. Notice that it satisfies $0 \leq L_{i}(t) \leq U_{i}(t) \leq 1$.

That is,

if $L_{i}(t)<X(t)<U_{i}(t) \quad$ then bystander $i$ takes action the following day

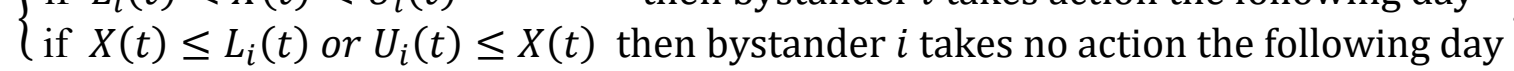

Subject to $0 \leq L_{i}(t) \leq U_{i}(t) \leq 1$, the upper triangle AOB in Figure 1 shows the area where a point $\left(L_{i}(t), U_{i}(t)\right)$ could exist. If the point $\left(L_{i}(t), U_{i}(t)\right)$ is near the vertex A of this triangle, lower threshold $L_{i}(t)$ is close to 0 and upper threshold $U_{i}(t)$ is close to 1 , so the active zone of this bystander $\left(L_{i}(t), U_{i}(t)\right)$ is quite wide. On the other hand, if the point $\left(L_{i}(t), U_{i}(t)\right)$ is near the line segment BO of this triangle, where $L_{i}(t)$ and $U_{i}(t)$ are fairly close, and the active zone $\left(L_{i}(t), U_{i}(t)\right)$ are extremely narrow. 
20-22 March, 2020

Berlin, Germany $10^{\text {th }}$ International Conference on Humanities, Psychology \& Social Sciences

Figure 1: The active zone of bystander (upper triangle AOB)

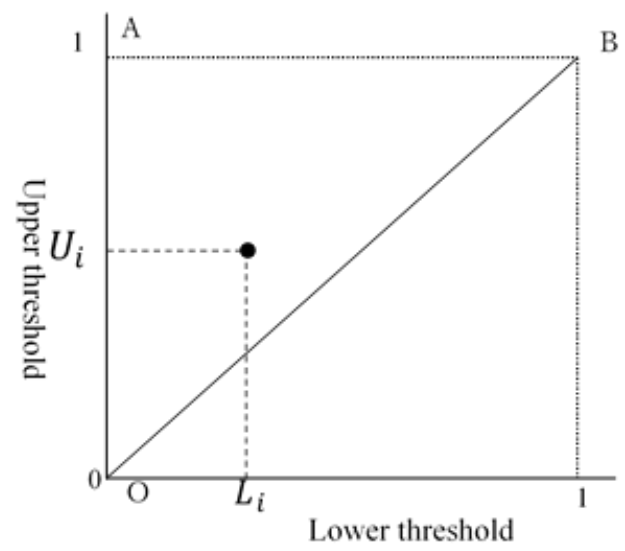

As shown in Figure 2, set the two parameters $a$ and $b$ in the range of $0 \leq a \leq b \leq 1$. A straight line passing through the point $(0, a)$ and parallel to the line segment $\mathrm{BO}$ and a straight line passing through the point $(0, b)$ and parallel to the line segment $\mathrm{BO}$ are drawn. It is assumed that the point $\left(L_{i}(t), U_{i}(t)\right)$ is uniformly distributed in the area between these two straight lines, that is, the area shown by the diagonal lines in Figure 2. This area is simply called Bystander Distribution Area. The similar mathematical model in which each person has two thresholds, upper and lower, and acts within that range, is used in literatures (Ishii, 1987 and Matsuda, 1996) etc. as a model renresenting enidemic nhenomena.

Figure 2: Bystander Distribution Area

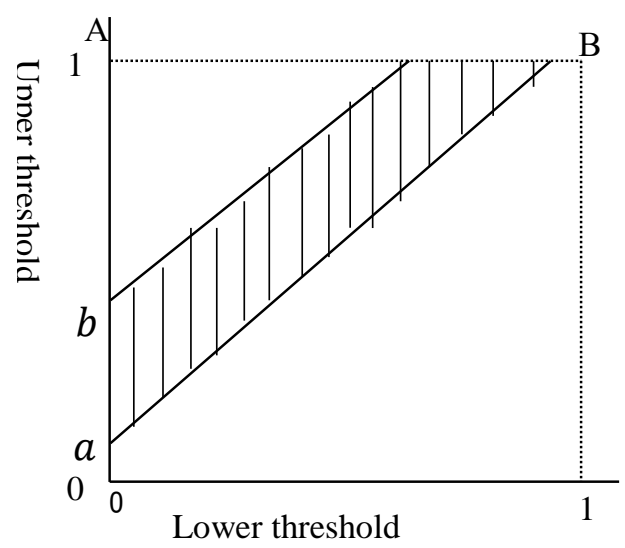

The active zone changes over time. When the active rate $X(t)$ exceeds 0.5 , the active zone of each bystander expands by $\Delta(t) r$ on both sides. If it is less than 0.5 , both sides of the active zone will shrink by $\Delta(t) r$. Here, $r$ is a non-negative parameter that controls fluctuation of the active zone, and $\Delta(t)$ is a basic fluctuation amount determined by a cubic function expressed by the following equation (2).

$$
\Delta(t)=x^{3}-1.5 x^{2}+2.5 x-1 \cdots
$$

As a result, as shown in Figure 3, the basic fluctuation amount $\Delta(t)$ of the active zone varies in the range of $-1 \leq \Delta(t) \leq 1$, and when $X(t)=1$, the value of $\Delta(t)$ is 1 ; when $X(t)=0$, the value of $\Delta(t)$ is -1 . The active zone $\left(L_{i}(t), U_{i}(t)\right)$ on the day $t$ changes to the active zone 
20-22 March, 2020

Berlin, Germany $10^{\text {th }}$ International Conference on Humanities, Psychology \& Social Sciences

$\left(L_{i}(t+1), U_{i}(t+1)\right)$ on the day $(t+1)$. The active zone $\left(L_{i}(t+1), U_{i}(t+1)\right)$ of the bystander $i$ on the day $(t+1)$ is determined by the following formulas (3) and (4).

$$
\begin{aligned}
& \left(L_{i}(t+1)\right)=\left\{\begin{array}{cc}
L_{i}(t)-\Delta(t) r\left(\text { for } 0 \leq L_{i}(t)-\Delta(t) r\right) \\
0 \quad & \left(\text { for } L_{i}(t)-\Delta(t) r<0\right)
\end{array}\right. \\
& \left(U_{i}(t+1)\right)=\left\{\begin{array}{cc}
U_{i}(t)+\Delta(t) r\left(\text { for } U_{i}(t)+\Delta(t) r \leq 1\right) \\
1 \quad\left(\text { for } 1<U_{i}(t)+\Delta(t) r\right)
\end{array}\right.
\end{aligned}
$$

Figure 3: The basic fluctuation amount $\Delta(t)$ of the active zone

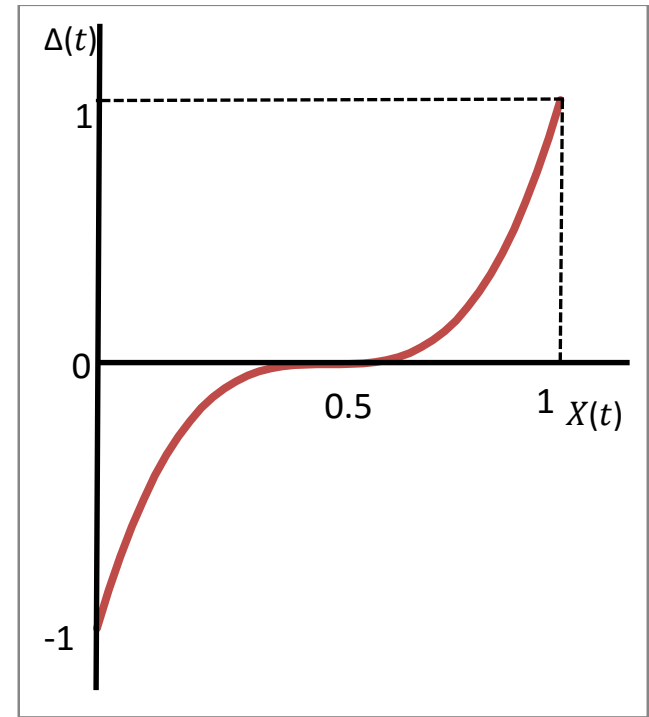

\section{Simulation experiment and its analysis}

A simulation experiment was performed to analyse the behaviour of bystanders. Multi-agent simulation (MAS) was used for the simulation experiments. The simulation platform is artisoc4.0. We deal with both cases where the active zone of the bystander fluctuates $(r=$ $0.005)$ and does not fluctuate $(r=0)$, for 1000 students. The parameter $a$ is set to 0 to 0.9 and $b$ is set to a value of 1 to $a+0.1$. A simulation experiment was performed by changing the values of $a$ and $b$ in units of 0.1 . The simulation goes on 30 days and the initial value of $X(t)$ is $X(0)=0.01$.

Figure 4 shows the change of the active rate $X(t)$ when $a=0$ and $r=0$. The horizontal axis shows the number of days. As the number of days progresses, the active rate $X(t)$ gradually increases and converges to a certain value. When $b=1$ and $b=0.9$, the values of $X(t)$ barely exceed 0.5. Otherwise, the values of $X(t)$ converge to values less than 0.5 . On the other hand, 
20-22 March, 2020

\section{Berlin, Germany}

\section{$10^{\text {th }}$ International Conference on Humanities, Psychology \& Social Sciences}

in the case of $a=0$ and $r=0.005$ (Figure 5), the values of $X(t)$ do not increase and immediately fall to zero without rising, except for the case of $b=1$. Even when $b=1$, the value of $X(t)$ rises but then gradually falls. The reason for this phenomena may be explained as follows. The maximum value of $X(t)$ is less than 0.5 , so that the active zone of each person is reduced, and thereby the value of $X(t)$ is decreased. This process is repeated, as a result, the negative feedback occurs.

Similar results were obtained when $a=0.1,0.2$ and $r=0,0.005$. However, when $a=0.2$, $r=0.005$, slight difference is shown in Figure 6 , that is, when $\mathrm{b}=1$, the value of $X(t)$ vibrates up and down around day 5 to day 10. This is because the active rate rises at once and exceeds the upper threshold of the active zone, the number of people who stop action increases, and as a result, the active rate decreases. Conversely, the active rate decreases at once and exceeds the lower threshold of the active zone, the number of people who take action increases, and as a result, the active rate increases. Such vibration phenomena are also seen in Figure 7 to Figure 11, Figure 13, and Figure 14.

In Figure 6, this vertical vibration subsides immediately, and at this point, the active rate has exceeded 0.5 , which means that positive feedback is occurring.

In the following, we will look at other cases. In most cases, when $r=0.005$, the active ratio did not increase, but when $r=0$, the value of $X(t)$ increases smoothly in all cases. In an actual situation, bystanders may not cooperate with the minority. Therefore, non-zero values of $r$ supposed to be better than zero values of $r$ for actual bystander behaviour.

When the value of $\mathrm{r}$ is larger than 0.005 , the situation where the value of $X(t)$ does not rise occurs more extremely, so in this paper, the case of $r=0.005$ is adopted.

Figure 7 shows the case where $a=0.3$ and $r=0.005$. Here, the periodic behaviour appeared clearly. This was the same when $a=0.4$. In addition, in Figure 7, among the seven graphs, in three graphs $(b=0.8,0.9,1)$ in which the value of $b$ is large, the value of $X(t)$ rises and does not fall to 0 . It is considered that the value of $b$ contributes to the increase in the value of $X(t)$. When $r=0.005$ and $a=0.5 \sim 0.8$, there are cases where $X(t)$ fall to zero. In the case of surviving without dropping to zero, chaotic behaviours are observed when $a=0.5$ (Figure 8 ) or $a=0.6$ (Figure 9).

When $a=0.7, X(t)$ fall to zero in both cases $r=0$ (Figure 10) and $r=0.005$ (Figure 11). On the other hand, when $a=0.8, r=0$ (Figure 12), $X(t)$ fall to zero, and chaotic behaviour is occurred for $r=0.005$ (Figure 13), $b=1$.

In Figure 14, the graph shows a periodic behaviour, but its amplitude gradually narrows and the median of the amplitude gradually increases. This may be due to positive feedback.

Another case of positive feedback is seen in Figure 7 when $X(t)$ is 0.5 or more, however, the effect of positive feedback is subtle. 
20-22 March, 2020

Berlin, Germany $10^{\text {th }}$ International Conference on Humanities, Psychology \& Social Sciences

Figure 4:Transition of the active rate $X(t)(a=0, r=0)$

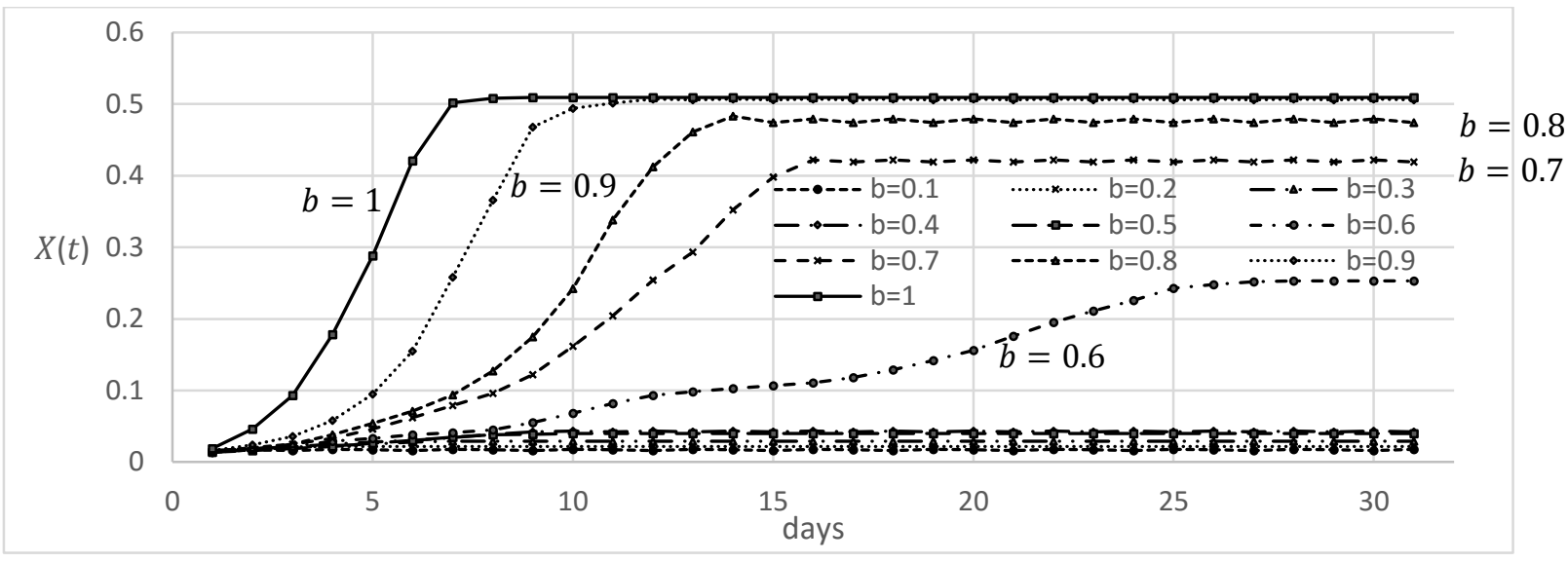

Figure 5: Transition of the active rate $(t)(a=0, r=0.005)$

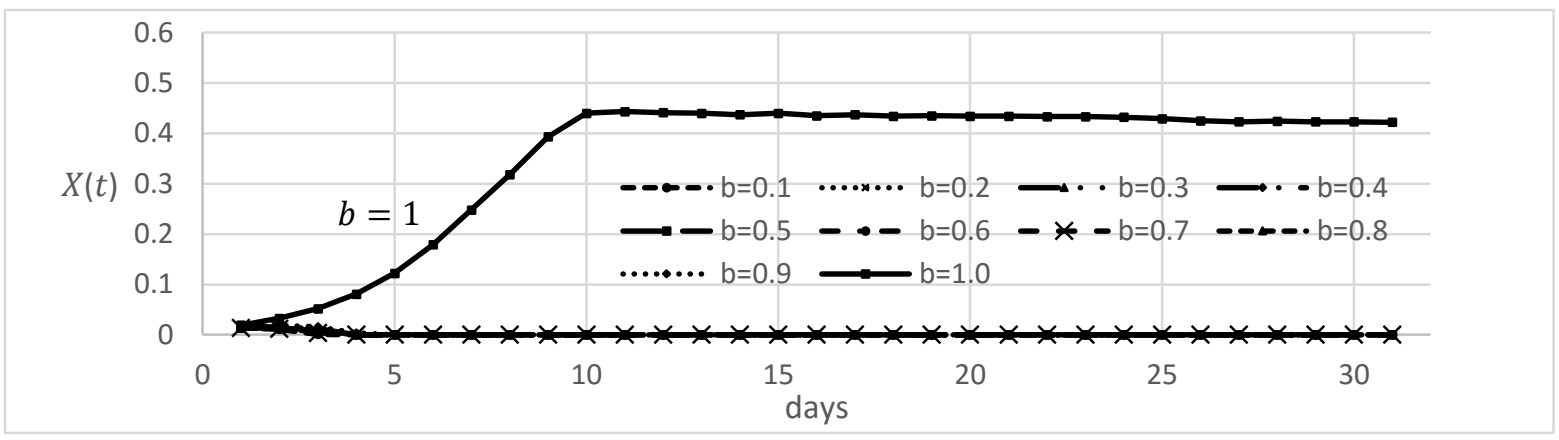

Figure 6: Transition of the active rate $(t)(a=0.2, r=0.005)$

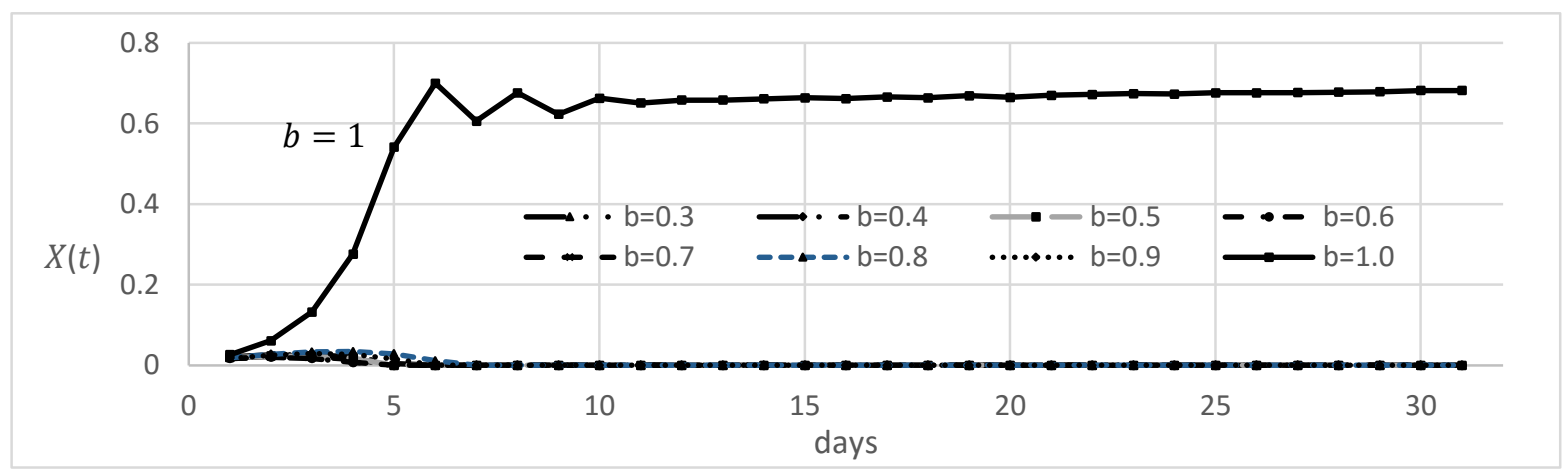




\section{HPSCONF}

20-22 March, 2020

Berlin, Germany $10^{\text {th }}$ International Conference on Humanities, Psychology \& Social Sciences

Figure 7: Transition of the active rate $(t)(a=0.3, r=0.005)$

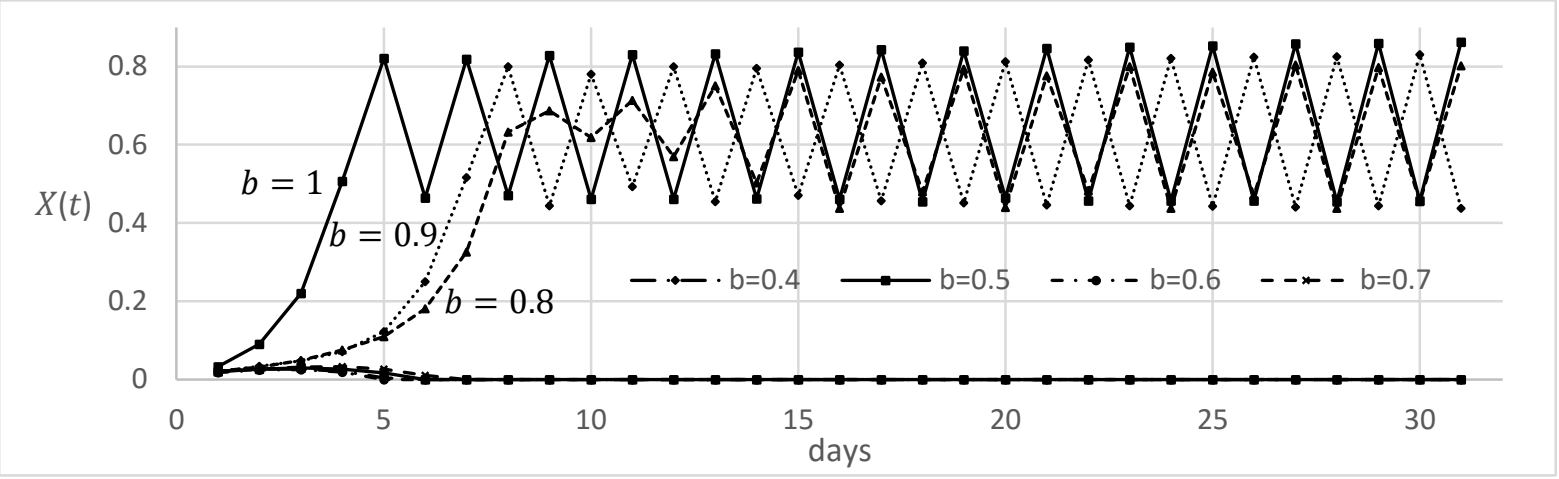

Figure 8: Transition of the active rate $(t)(a=0.5, r=0.005)$

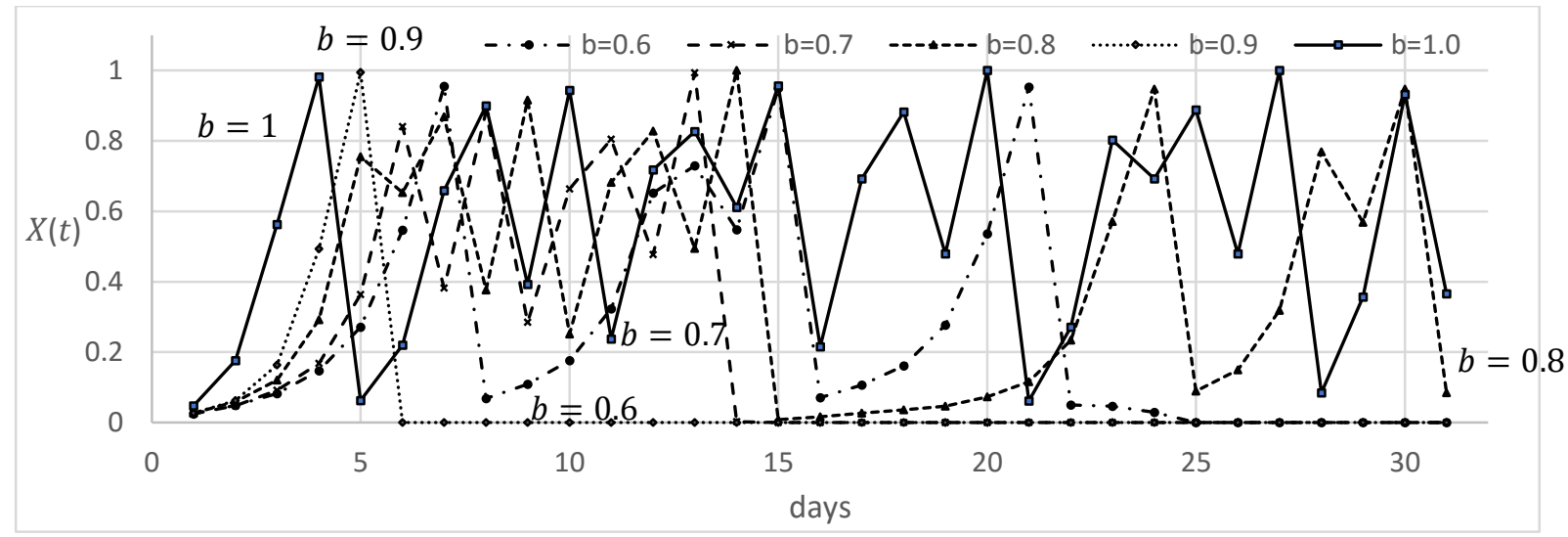

Figure 9: Transition of the active rate $(t)(a=0.6, r=0.005)$

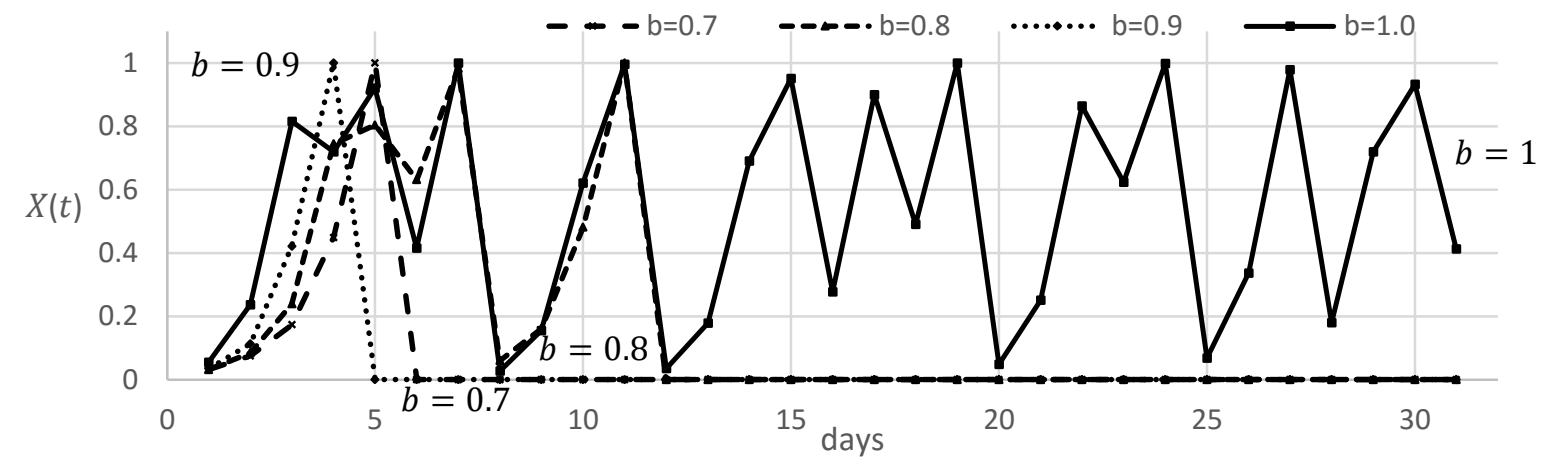

Figure 10: Transition of the active rate $(t)(a=0.7, r=0)$ 
20-22 March, 2020

\section{Berlin, Germany}

$10^{\text {th }}$ International Conference on Humanities, Psychology \& Social Sciences

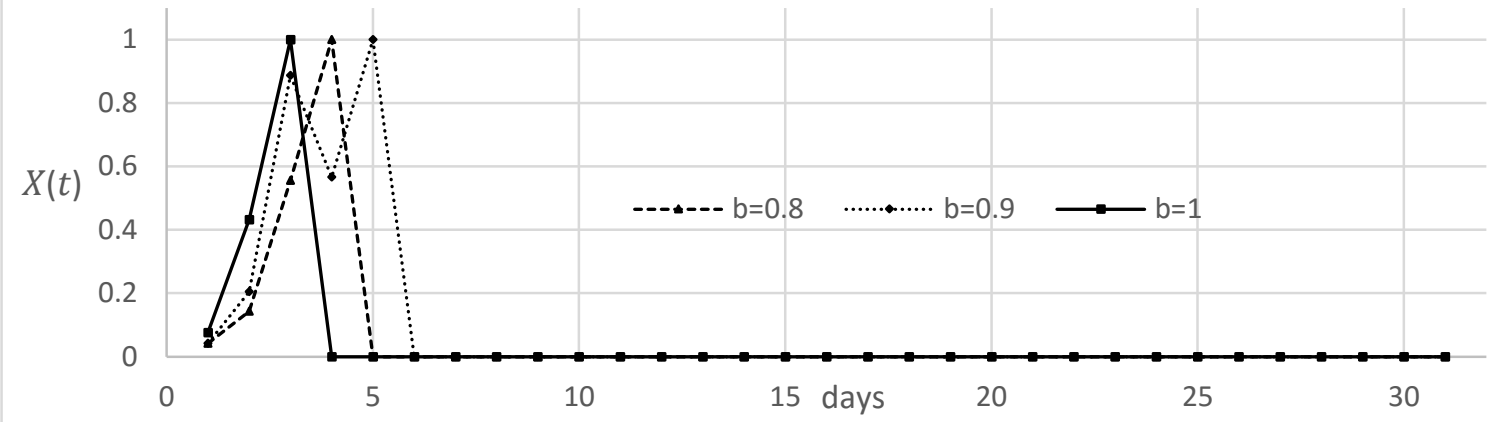

Figure 11: Transition of the active rate $(t)(a=0.7, r=0.005)$

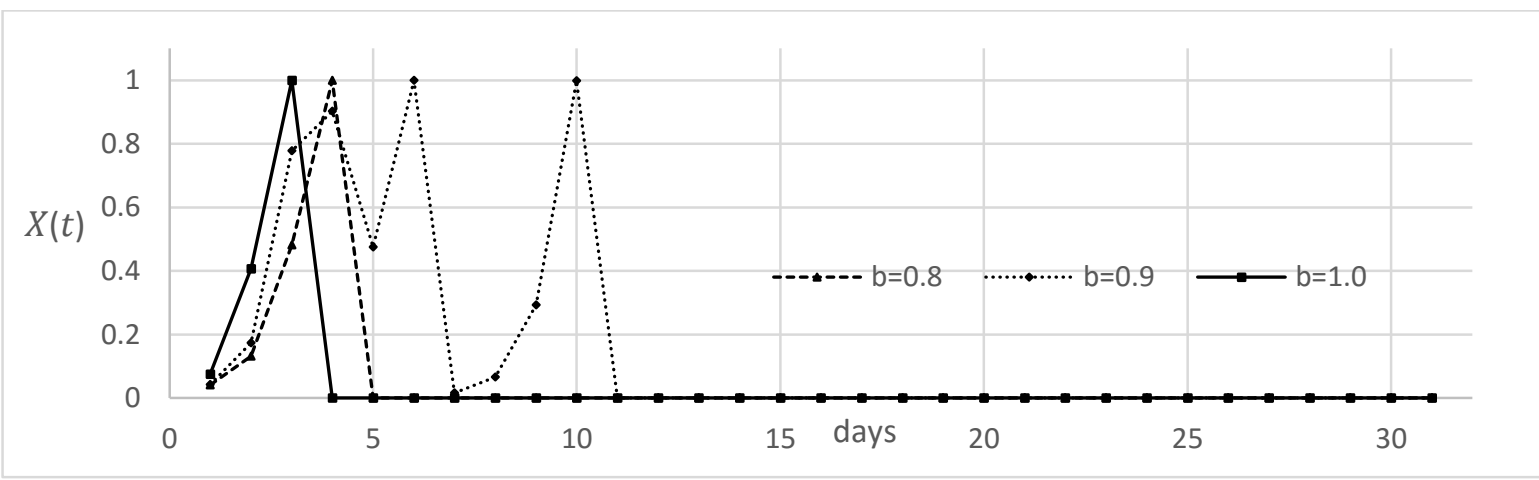

Figure 12: Transition of the active rate $(t)(a=0.8, r=0)$

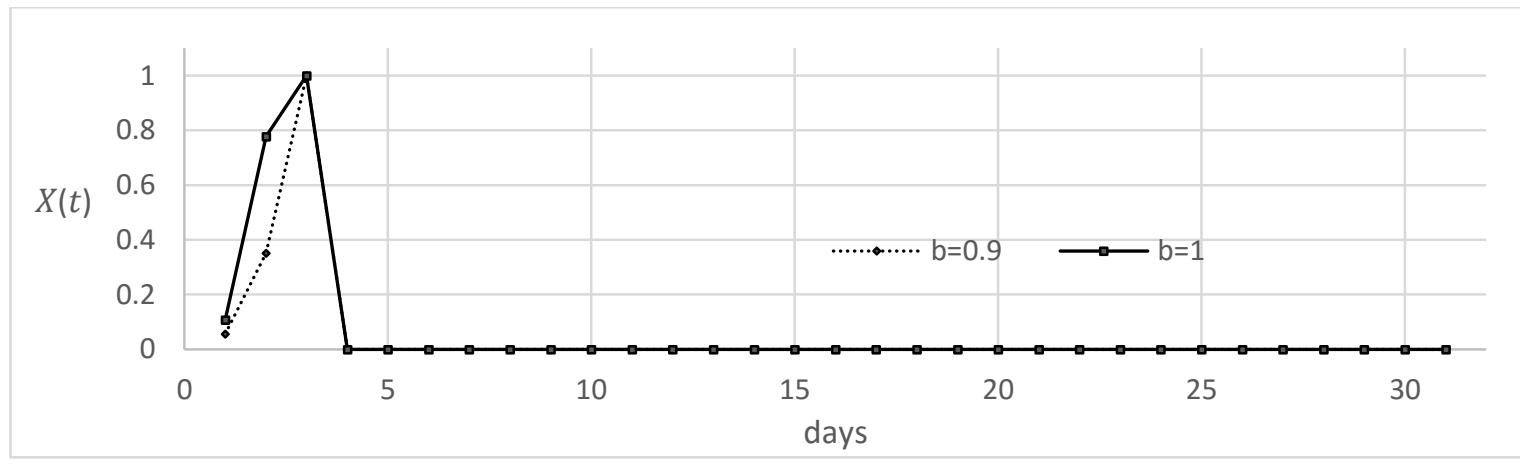

Figure 13: Transition of the active rate $(t)(a=0.8, r=0.005)$

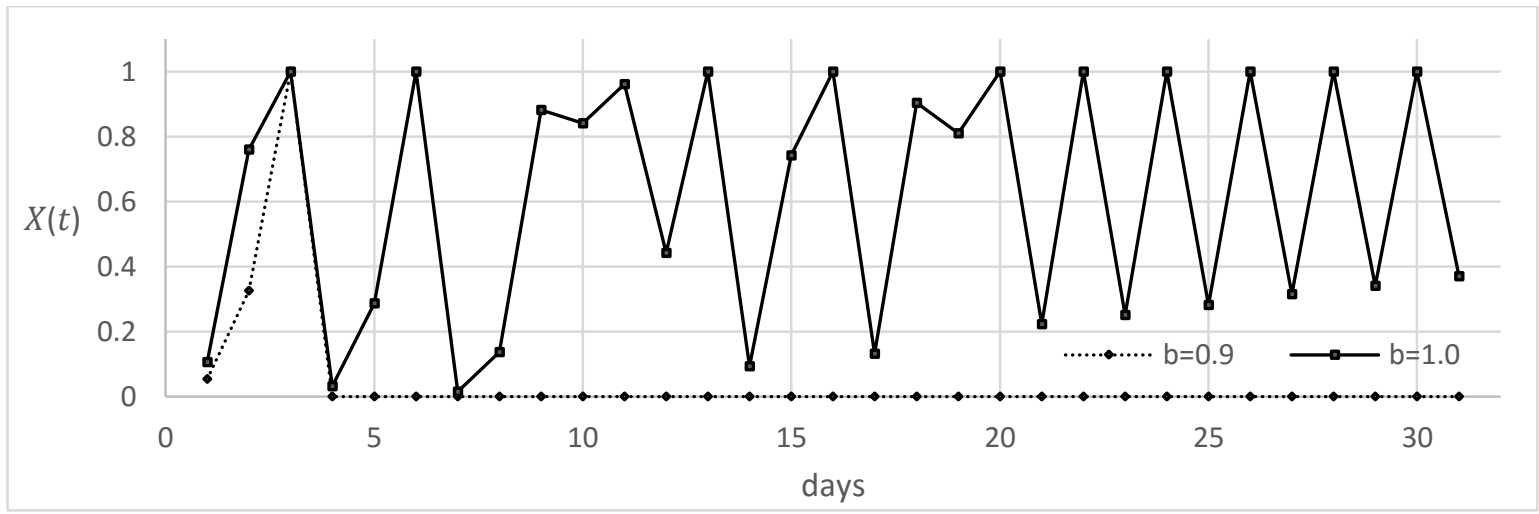


Figure 14: Transition of the active rate $(t)(a=0.9, r=0.005)$

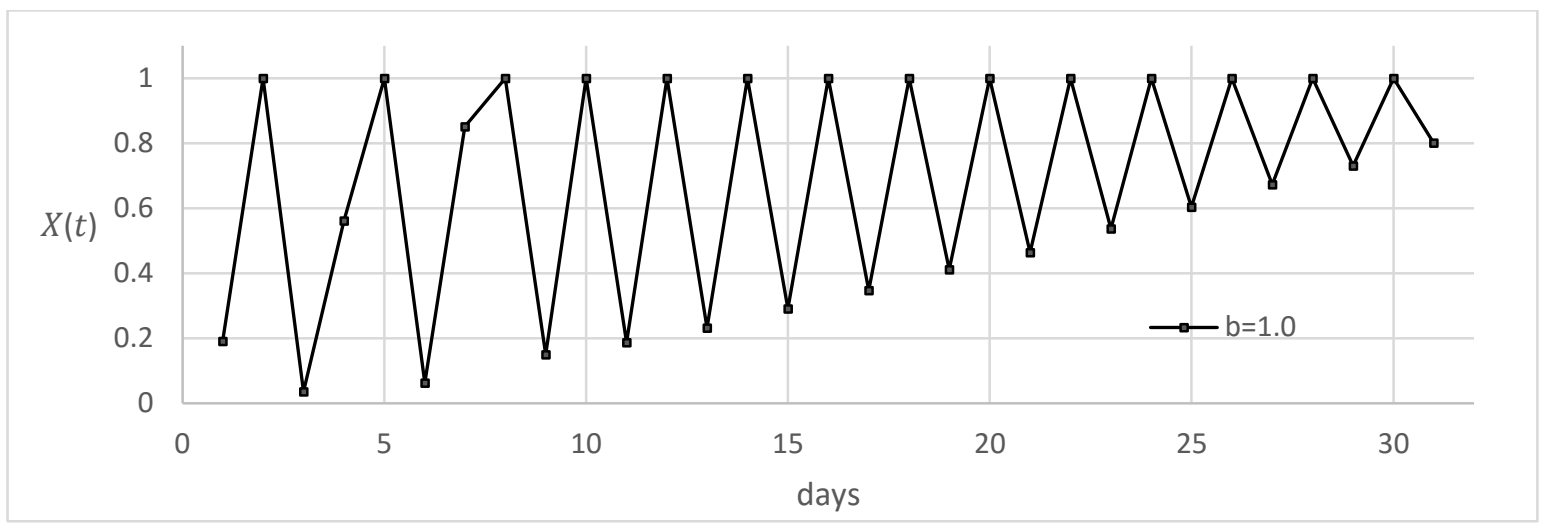

\section{Changes in bystander behaviour owing to educational policies}

In this section, we examine the effects of two educational policies by changing the Bystanders Distribution Area defined by $a=0.2$ and $b=0.7$.

The first educational policy is to educate people with narrow active zones and raise their motivation. In other words, we aim to change Bystanders Distribution Area to the area corresponding to $a=0.3$ and $b=0.7$. The second educational policy is to increase the number of people in the active zone and to activate more highly conscious people. In other words, we aim to change Bystanders Distribution Area to the area corresponding to $a=0.2$ and $b=0.8$. Figure 15 shows the results, but both educational policies have no noticeable effect. However, when both policies are performed simultaneously ( $a=0.3, b=0.8)$, the value of $X(t)$ jumps up greater than 10 times.

Figure 16 shows the results of a similar experiment in which the increments of the values of $a$ and $b$ were each set to 0.2 . Here, a remarkable effect appears when only $a$ is raised, and the effect is almost equivalent to the effect when both $a$ and $b$ are raised at the same time.

Next, we compare the policy of increasing only $a$ by 0.1 with the policy of increasing the only $b$ by 0.1 based on $a=0.3$ and $\mathrm{b}=0.8$. Figure 17 shows the results. It can be seen that both policies have little effect on the whole except that the rise is slightly quicker. If both policies are implemented at the same time, the start-up is a little faster.

Figure 18 shows the results of examining the policy of increasing $a$ and $b$ by 0.2 each in the same area. When $a$ is increased by 0.2 , unstable chaotic behaviour is noticeable. When $b$ is increased by 0.2 , the behaviour is stable and periodic, but it is shown that the effect of raising $b$ is not so clear. If both policies are implemented at the same time, chaotic behaviour will be seen, although the rise will be faster. The change to chaotic behaviour seems to be due to the increase in the value of $a$. 


\section{HPSCONF}

20-22 March, 2020

\section{Berlin, Germany}

$10^{\text {th }}$ International Conference on

Humanities, Psychology \& Social Sciences

Figure 15: Changes in the active rate when $a$ and $b$ are each increased by 0.1 based on $a=0.2$ and $b=0.7$

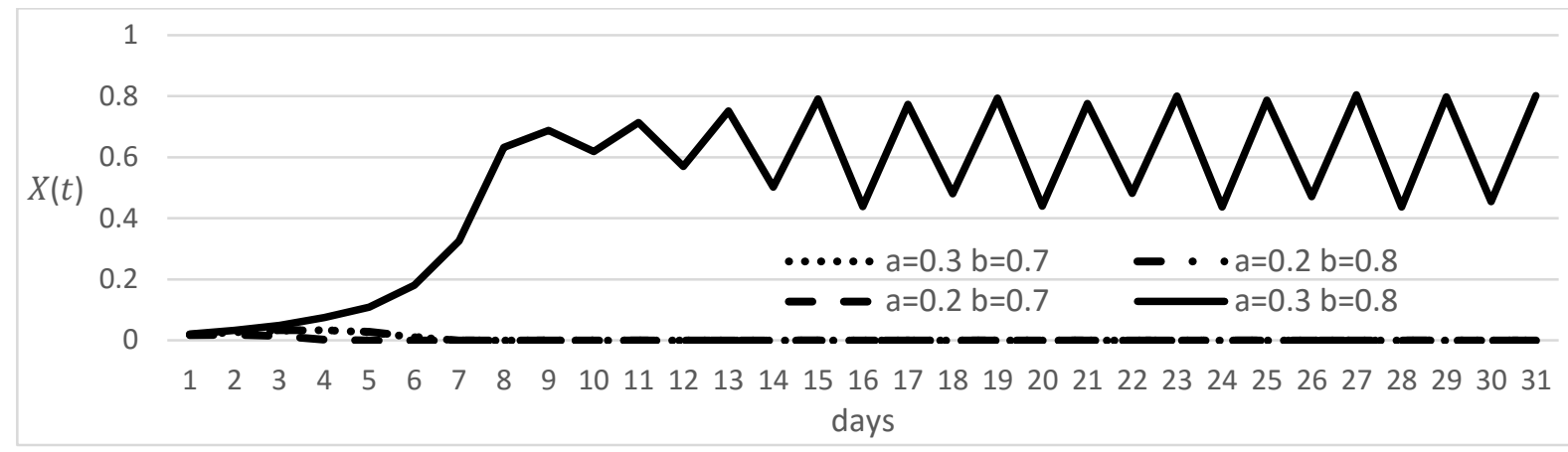

Figure 16: Changes in the active rate when $a$ and $b$ are each increased by 0.2 based on $a=0.2$ and $b=0.7$

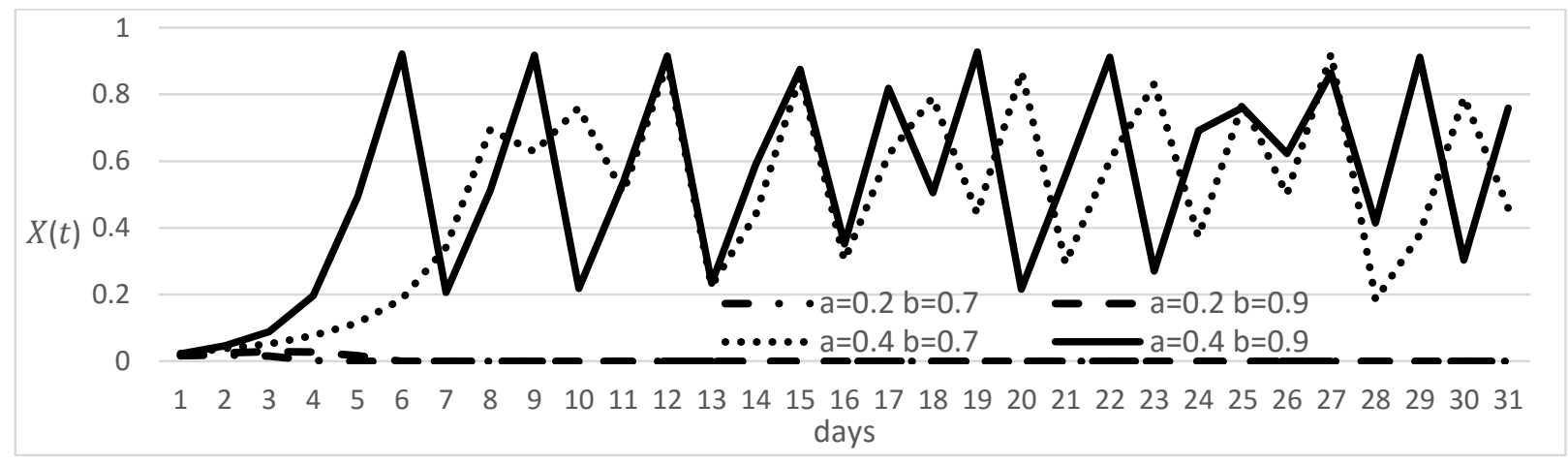

Figure 17: Changes in the active rate when $a$ and $b$ are each increased by 0.1 based on $a=0.3$ and $b=0.8$

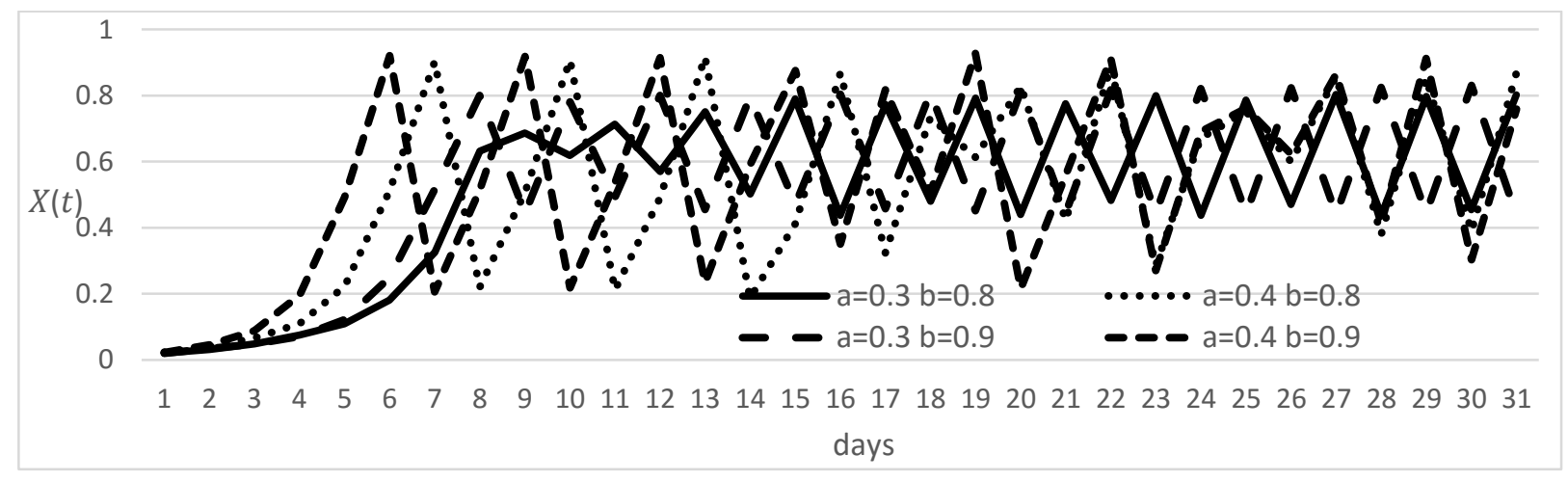


Figure 18: Changes in the active rate when $a$ and $b$ are each increased by 0.2 based on $a=0.3$ and $b=0.8$

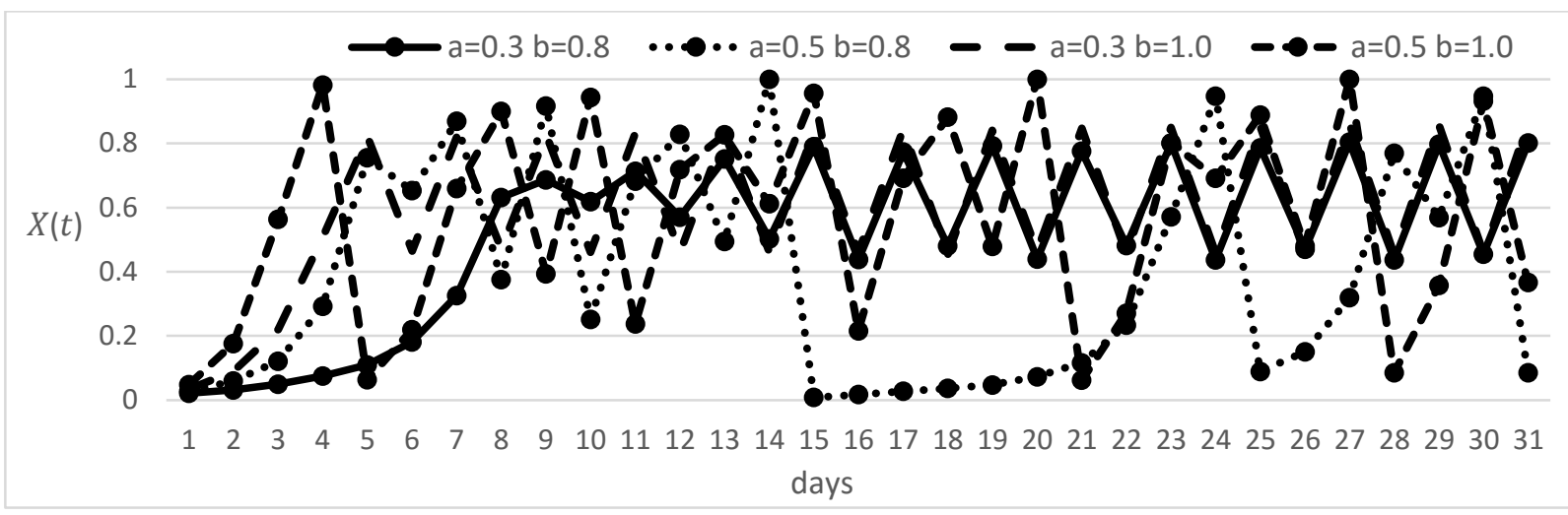

Next, let's focus only on the maximum value of $X(t)$ for $t=1, \ldots, 31$. We write this as $X_{\max }=X_{\max }(a, b)$. Table 1 shows the change of $X_{\max }$ when $a$ and $b$ are changed. Figure 19 illustrates this data.

From Table 1, it can be seen that $X_{\max }$ monotonically increases when the value of $b=1$ is fixed and the value of $a$ is increased. This is because, for example, when $a=a_{1}$ rises to $a=$ $a_{2}\left(a_{1}<a_{2}\right)$, there are no members whose active zone is included in $\left(a_{1}, a_{2}\right)$ and there are only members with high motivation whose active zones are in the interval $\left(a_{2}, 1\right)$.

According to Table 1 , when $b=0.1$ to $0.4, X_{\max }$ remains below 0.1 , but when $b=0.8$ and 0.9 (this is called the first group), it is greatly increased at $a=0.3$ (See data with the thick frame in Table 1). When $b=0.5,0.6,0.7$ (this is called the second group), the value of $X_{\max }$ increases greatly at $a=0.4$ (See data with the thick frame in Table 1). In both the first group and the second group, $X_{\max }$ suddenly increases at a certain value of a to a value close to $X_{\max }$ when $b=1$. In other words, when $b<1$, when trying to raise the level of members with low motivation, the effect may not appear immediately, but if you continue without giving up, suddenly the effect will become enormous. Until the effect appears, the value of $X_{\max }$ is much lower than $X_{\max }$ when $b=1$, which is shown in Figure 19.

From the above, it can be seen that the most important factor to obtain high $X_{\max }$ is whether there are high motivation members in the group or not. Besides, even if very highly motivated members do not exist, it is possible to obtain high $X_{\max }$ by raising the value of $a$ through education. However, as can be seen from Table 1, when only members with very low motivation, such as $b \leq 0.4$, the increase of $X_{\max }$ cannot be expected since the value of $a$ cannot be increased beyond the values of $b$

Table 1 Transition of $\boldsymbol{X}_{\max }$ when parameters $\boldsymbol{a}$ and $\boldsymbol{b}$ are changed

\begin{tabular}{rrrrrrrrrrr}
\hline & $b=0.1$ & $b=0.2$ & $b=0.3$ & $b=0.4$ & $b=05$ & $b=0.6$ & $b=0.7$ & $b=0.8$ & $b=0.9$ & $b=0.1$ \\
\hline$a=0$ & 0.014 & 0.016 & 0.015 & 0.016 & 0.014 & 0.014 & 0.014 & 0.015 & 0.019 & 0.443 \\
$a=0.1$ & & 0.021 & 0.022 & 0.022 & 0.017 & 0.015 & 0.016 & 0.031 & 0.025 & 0.602 \\
$a=0.2$ & & & 0.022 & 0.026 & 0.023 & 0.02 & 0.019 & 0.034 & 0.029 & 0.7 \\
$a=0.3$ & & & & 0.026 & 0.029 & 0.025 & 0.033 & 0.804 & 0.831 & 0.863 \\
\hline
\end{tabular}


20-22 March, 2020

Berlin, Germany $10^{\text {th }}$ International Conference on Humanities, Psychology \& Social Sciences

\begin{tabular}{rlrrrrrr}
\hline$a$ & $=0.4$ & 0.777 & 0.867 & 0.922 & 0.917 & 0.928 & 0.941 \\
$a$ & $=0.5$ & & 0.955 & 0.994 & 1 & 1 & 0.995 \\
$a$ & $=0.6$ & & & 1 & 1 & 1 & 1 \\
$a$ & $=0.7$ & & & & 1 & 1 & 1 \\
$a$ & $=0.8$ & & & & & 1 & 1 \\
$a$ & $=0.9$ & & & & & & 1 \\
\hline
\end{tabular}

Figure 19: Transition of $X_{\max }$ when parameters $a$ and $b$ are changed

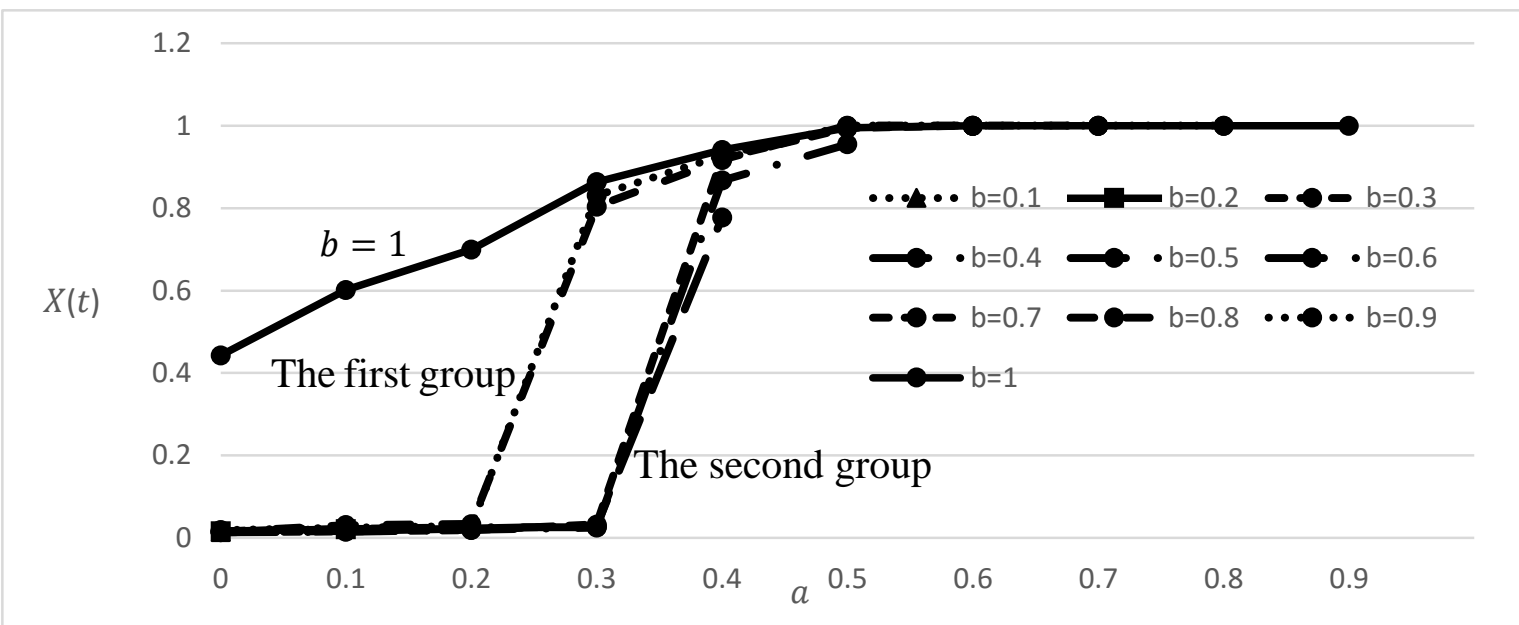

\section{Conclusion}

In this paper, we formulate a behaviour model of bystanders assuming that each bystander has its active zone. As a result, various types of bystanders' behaviour are observed depending on Bystanders Distribution Area. It is found that the presence of highly motivated members has a strong effect on all the bystanders. On the other hand, even if very highly motivated members do not exist, educating people with low motivation without giving up could be quite effective.

Besides, in this model, each person decides whether to act or not to act only based on the active rate. However, in real situations, people are strongly influenced by those who have strong ties to themselves. As a future research topic, it is necessary to deal with a model that considers the degree of connection between bystanders.

\section{Acknowledgment}

This work was supported by KAKENHI Grant Numbers 17K01282.

\section{References}

[1] Ishii, K. (1987). "Threshold Models of public opinion process," Sociological Theory and Methods, Vol.2, No.1, pp.15-28 (in Japanese). 
20-22 March, 2020

\section{Berlin, Germany}

$10^{\text {th }}$ International Conference on Humanities, Psychology \& Social Sciences

[2] Matuda, K. (1996). "Phenomenon of Fashion and Vector Threshold Models," Sociological Theory and Methods, Vol.11, No.2, pp.113-128 (in Japanese).

[3] Morita, Y. (2010). Ijime to ha nanika [What Is Bullying], Tokyo, Japan: Chuko Shinsho (in Japanese).

[4] The Ministry of Education, Culture, Sports, Science and Technology (MEXT) (2013), Heisei 25nendo ijimeboushitaisaku suishinhou [Bullying Prevention Measures Promotion Act] [Online] (in Japanese) Available:

http://www.mext.go.jp/a_menu/shotou/seitoshidou/1337219.htm

[5] MEXT, (December, 2014). Heisei 25nendo 'Jidouseito no mondaikoudounadoseitoshidoujou no shomondainikansuruchousakek-kanitsuite [Results of the 2013 Survey of Problem Behaviour of Students and Various Issues with Instructing Students] [Online]( in Japanese) Available:

http://www.mext.go.jp/b_menu/houdou/26/12/_icsFiles/afieldfile/2014/12/19/1354076_01_2 .pdf

[6] MEXT (October, 2018) Heisei 29nendo 'Jidouseito no mondaikoudounadoseitoshidoujou no shomondainikansuruchousakek-kanitsuite ["Results of the 2017 Survey of Problem Behaviour of Students and Various Issues with Instructing Stu-dents] [Online] (in Japanese) Available:

http://www.mext.go.jp/b_menu/houdou/30/10/_icsFiles/afieldfile/2018/10/25/1410392_1.pdf 